\title{
On the Performance Bounds of Practical Wireless Network Coding
}

\author{
Jilin Le*, John C.S. Lui* and Dah Ming Chiu ${ }^{+}$ \\ The Chinese University of Hong Kong \\ ${ }^{*}$ Computer Science \& Engineering Department \\ ${ }^{+}$Information Engineering Department \\ Email: \{jlle,cslui\}@cse.cuhk.edu.hk,dmchiu@ie.cuhk.edu.hk
}

\begin{abstract}
Network coding is an attracting technology that has been shown to be able to improve the throughput of wireless networks. However, there still lacks fundamental understanding on how network coding works under realistic scenarios. In this paper, we examine the performance of a recently proposed network coding system under a realistic wireless physical layer and practical random access mechanisms. We propose a key performance measure called "encoding number"-the number of packets that can be encoded via network coding in each transmission. We provide an upper bound on the encoding number for the general coding topology, and derive the average encoding number and system throughput for a general class of random access mechanisms. Based on the practical coding system, we also derive a tighter upper bound on the throughput gain for a general wireless network. Our results are of fundamental value for coding-related MAC/Routing protocol design and analysis.
\end{abstract}

Keywords: network coding, wireless networks, performance evaluation.

\section{Introduction}

Network coding has been shown to be able to improve the throughput of wireless networks. Recently, the authors of [5] proposed the practical coding system called COPE. COPE utilizes local coding opportunities around each node, and the basic coding scenarios are shown in Fig. 1. Consider the example in Fig.1(a), suppose node $S_{1}$ wants to transmit a packet $P_{1}$ to node $D_{1}$ via node $C$, while node $S_{2}$ wants to transmit $P_{2}$ to $D_{2}$ via node $C$. The dashed arrows $S_{1} \rightarrow D_{2}$ and $S_{2} \rightarrow D_{1}$ indicate that $D_{2}, D_{1}$ are within the transmission ranges of $S_{1}, S_{2}$ respectively. Therefore, $D_{1}, D_{2}$ can perform "opportunistic listening": when $S_{1}\left(S_{2}\right)$ transmits $P_{1}\left(P_{2}\right)$ to node $C$, node $D_{2}\left(D_{1}\right)$ can overhear the transmission of $P_{1}\left(P_{2}\right)$. Without network coding, node $C$ needs to transmit $P_{1}$ and $P_{2}$ separately. However, under COPE, node $C$ can broadcast an encoded packet $\left(P_{1} \oplus P_{2}\right)$ to both $D_{1}$ and $D_{2}$, then $D_{1}$ can decode $P_{1}$ by performing $P_{2} \oplus\left(P_{1} \oplus P_{2}\right)$, while $D_{2}$ can decode $P_{2}$ by performing $P_{1} \oplus\left(P_{1} \oplus P_{2}\right)$. Therefore, node $C$ delivers two packets worth of information using a single transmission so that $1 / 4$ of the bandwidth is saved. Another typical coding scenario is shown in Fig.1(b), where no opportunistic listening is required because each of the two source nodes are also destination nodes. Finally, Fig.1(c) shows a hybrid form of coding which combines the former two cases, namely, some packets for decoding are obtained via opportunistic listening while other packets are obtained by the fact that the node is the source of that packet. In this scenario, node $C$ can at most encode four packets together and save three transmissions.

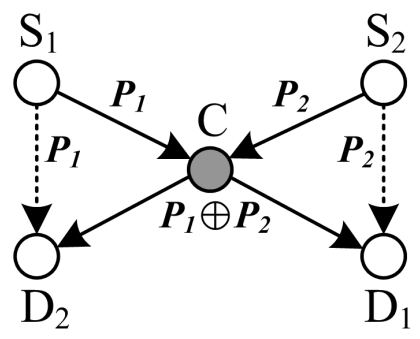

(a) Coding scenario with opportunistic listening.

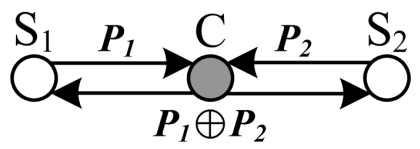

(b) Coding scenario without opportunistic listening.

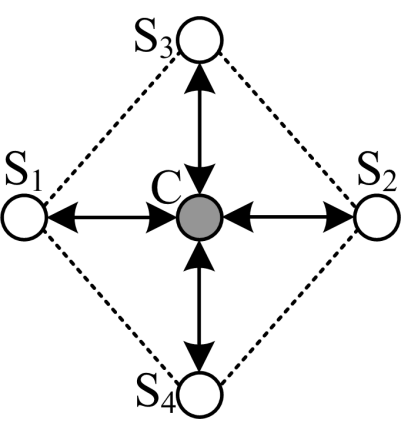

(c) Hybrid scenario.

Fig. 1. Basic scenarios of XOR coding under idealized link-scheduling.

We use the term encoding number to refer to the number of packets that can be encoded by a relay node (i.e., node $C$ in Fig.1) in each transmission. Intuitively, the higher the encoding number, the higher bandwidth efficiency and throughput can be achieved. One fundamental question is whether there is an upper bound on the encoding number for a general coding structure. While former work [5] assume that there can be infinite nodes around the relay node such that the encoding number is unbounded, we show that this number is upper bounded by a constant for a general coding structure (in Section II). As we will show in later sections, the upper bound of encoding number directly affects the highest throughput gain by the coding scheme, as well as other performance measures like throughput and packet loss ratio. 
Another important question that we address is how well the coding scheme works under random access link-scheduling mechanisms. For example, in Fig.1(a), if the link-scheduling is such that the transmitters always transmit following the cycle of $S_{1}, S_{2}, C, \cdots$ (or $\left.S_{2}, S_{1}, C, \cdots\right)$, then node $C$ can always encode two packets in each transmission and maximize the total throughput. However, if the link-scheduling is $S_{1}, C, S_{2}, C, S_{1}, C, \cdots$, then node $C$ cannot encode any packets. In practice, most of the wireless link-scheduling algorithms are probabilistic (due to the random access mechanism) in nature and non-coding-oriented, i.e., the potential coding opportunity may not be fully utilized. In Section III, we model how different random access mechanisms affect the encoding number. In particular, we formally characterize the interplay of throughput, buffer size and the random access mechanism used. Surprisingly, we find that the simple "equal access" mechanism outperforms other sophisticated mechanisms in most cases.

In Section IV, based on the analysis in a single coding structure, we then provide an upper bound on the throughput gain by COPE for a general wireless network, where there can be multiple concurrent coding structures.

In summary, the main contributions of this paper include:

- We derive an upper bound on the encoding number for general coding structures. This shows the contradiction to the assumption made in [5].

- We propose a methodology to obtain the average encoding number under a general class of random access mechanisms.

- We compare the performance of different random access mechanisms, and find the importance of buffer size on the coding performance.

- We formally prove the upper bound of throughput gain by the practical XOR coding system (COPE) for general wireless networks.

The paper is organized as follows. In Section II, we characterize the general coding structure, and provide an upper bound on the encoding number in any possible coding structures under COPE. In Section III, we use a stochastic model to examine the coding performance under various random access mechanisms. In Section IV, we analytically derive the upper bound of throughput gain for general wireless networks. In Section $\mathrm{V}$, we verify the analysis by simulation. In Section VI, we introduce the potential applications of our results and the future work. In Section VII, we present the related work and Section VIII concludes.

\section{Coding Structure: Characterization and Properties}

\section{A. Assumptions and notations}

We first state the necessary assumptions and notations that will be used throughout this paper. We consider a stationary wireless network, where the nodes operate at single channel and half-duplex mode, that is, the bandwidth resource is timeshared like DCF in 802.11.
We focus on the COPE coding system. A "coding node" is the node which encodes packets for several flows, e.g., node $C$ in Fig.1(a) to 1(c) is a coding node. "Coding flows" are flows that transmit via a coding node and their packets have the opportunity to be encoded (e.g., flow $S_{1}-D_{1}$ and $S_{2}-D_{2}$ in Fig. 1(a) and 1(b)). A "coding structure" includes one coding node as well as the one-hop predecessor nodes and the onehop successor nodes of the associated coding flows. In general, there can be $n \geq 2$ coding flows within a coding structure. Clearly the encoding number, or flows that can be processed via network coding, is at most $n$ in one coding structure. Under COPE, when a coding node decides to use the XOR coding, then we say that a coding scheme is applied, otherwise, a noncoding scheme is used.

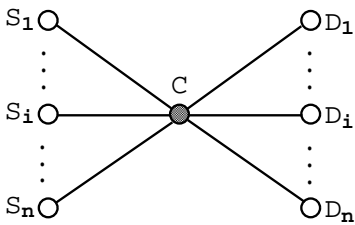

(a) Logical view.

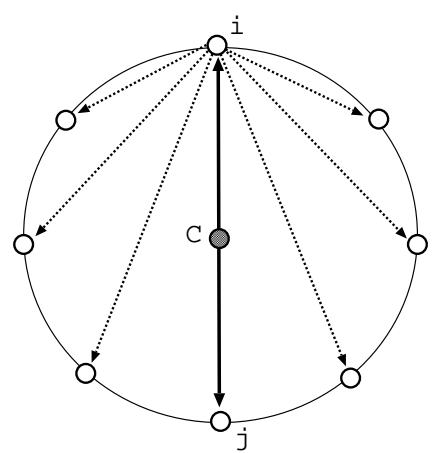

(b) Physical topology.
Fig. 2. (a) Logical and (b) physical representation of a coding structure.

Clearly there are certain geometrical constrains in a coding structure, which will be explored in more details in Section II-C, to ensure proper encoding and decoding. For the ease of presentation and analysis, we first show the "logical view" of a coding structure in Fig. 2(a), where $n$ two-hop coding flows intersect at the coding node $C$. One possible physical representation ${ }^{1}$ of this logical coding structure is shown in Fig. 2(b): there are $n$ nodes evenly spaced apart along a circle, the coding node $C$ is at the center of the circle. Each node along the circle, say node $i$, has its corresponding node $j$, and the segment $|i j|$ traverses node $C$ (i.e., $|i j|$ is a diameter of the circle). We assume that the transmission of node $i$ can be successfully received by all nodes along the circle except for node $j$. Each source node chooses its corresponding node as its destination, and all coding flows are relayed by node $C$ at the center. Therefore, in this symmetric structure, if we let each source node along the circle transmit one packet to node $C$ first, then node $C$ can encode all these $n$ packets and broadcast the encoded packet to all destination nodes along the circle. Each destination node can perform proper decoding because it has already obtained the other $n-1$ packets, either by opportunistic listening or due to the fact that it is the source of that packet.

\footnotetext{
${ }^{1}$ Please note that there can be other possible topologies, we choose this one here only because it can cover the general $n \geq 2$ cases.
} 


\section{B. Optimum Throughput in a Coding Structure}

Clearly, all the transmitters in a coding structure are within a single interference range. We denote the total channel bandwidth by $B$. The total throughput in a coding structure is the sum of end-to-end throughput of all its coding flows. Then the optimum total throughput for the non-coding scheme and coding-scheme can be achieved, when the conditions described in the following lemmas are met respectively.

Lemma 1 Under the "non-coding scheme", the optimum total throughput is achieved when "flow rate conservation" is ensured at the relay node (i.e. node C). In other words, the total bandwidth allocated to node $S_{1}, \ldots, S_{n}$ should be equal to the bandwidth allocated to node $C$. When this condition is met, the optimum total throughput under the non-coding scheme is $B / 2$.

Proof: Let $\lambda_{i}(i=1, \ldots, n)$ denote the draining rate of node $S_{i}$, and let $\mu$ denote the draining rate of node $C$. The total end-to-end throughput is equal to $\mu$. Obviously we have $\mu \leq \sum_{i=1}^{n} \lambda_{i}$. Because all nodes are within single interference range, we have $\sum_{i=1}^{n} \lambda_{i}+\mu \leq B$. Therefore, $\mu$ is at most $B / 2$, when $\mu=\sum_{i=1}^{n} \lambda_{i}$.

Lemma 2 Under the "coding scheme", the optimum total throughput is achieved when: 1) the transmission schedule follows some cyclic pattern like $S_{1}, S_{2}, \cdots, S_{n}, C$, such that the encoding number is maximized in each transmission; 2) equal bandwidth allocation to all $S_{1}, \ldots, S_{n}$ and $C$. When these conditions are met, the optimum total throughput under the coding scheme is $n B /(n+1)$.

Proof: Let $\lambda_{i}(i=1, \ldots, n)$ denote the draining rate of node $S_{i}$, and let $\mu$ denote the draining rate of node $C$. The total end-to-end throughput is equal to $\tau \mu$, where $\tau$ is the average encoding number of the coding node. Obviously this number is at most $n$. Similar to proof of Lemma 1, we have $\tau \mu \leq \sum_{i=1}^{n} \lambda_{i}$ and $\sum_{i=1}^{n} \lambda_{i}+\mu \leq B$. Obviously the maximum total throughput is obtained when $\tau=n$, which means all nodes must have equal draining rate, and the transmission schedule must follow the cyclic pattern like $S_{1}, S_{2}, \cdots, S_{n}, C$ to let the coding node encode $n$ packets in each transmission.

One should note that while the optimal throughput for noncoding scheme is a constant, the optimal throughput for coding scheme is crucially dependent on $n$, the number of coding flows in the coding structure, which is also the maximum encoding number in this coding structure.

\section{The Upper Bound of Maximum Encoding Number}

As discussed before, $n$, the number of coding flows within a coding structure, is the maximum encoding number and directly affects the optimal throughput of the coding scheme.
In [5], the authors assume that $n$ can be arbitrarily large. However, we will show that under a realistic wireless setting, $n$ is indeed bounded. The main reason for this upper bound is the geometrical constraints associated with opportunistic listening and two-hop relaying.

Consider a coding structure with $n>2$ coding flows. There are $n$ receivers located within the transmission range of the coding node. For each receiver, say receiver $D_{i}$, it has to decode its own packet from the XOR combination of $n$ packets. In other words, it must have already obtained the other $n-1$ packets either by (a) it has transmitted that packet or (b) it has overheard that packet by opportunistic listening. Note that for $n>2$ coding flows, there must be some opportunistic listening involved.

Suppose receiver $D_{i}$ gets packet $P_{j}$ (which is destined to receiver $D_{j}$ ) by opportunistic listening. Let $V_{j}^{i}$ denote the transmitter for this opportunistic listening, then $D_{i}$ must be within the transmission range of $V_{j}^{i}$ whereas $D_{j}$ must be outside the transmission range of $V_{j}^{i}$. Having this in mind, let $|A B|$ denote the distance between node $A$ and $B$, then we must have $\left|V_{j}^{i} D_{i}\right| \leq r$ and $\left|V_{j}^{i} D_{j}\right| \geq r+\delta$, where $r$ is the reliable transmission range of node $V_{j}^{i}$, and $\delta$ is a positive constant characterizing the distance gap between "reliable transmission" and "unreliable transmission". In other words, we say that if $|A B| \leq r$ then node $B$ can successfully receive node $A$ 's transmission with high probability, while if $|A B| \geq r+\delta$ then node $B$ can only receive node $A$ 's transmission with a very low probability.

Let us illustrate the concept of $r$ and $\delta$ in wireless networks. In [13], the authors derived the successful reception probability $(P)$ as a function of distance $(x)$ between a transmitter and a receiver under the log normal shadow fading model. In particular, $P(x)$ can be approximated as:

$$
P(x)= \begin{cases}1-\left(\left(\frac{x}{R}\right)^{2 \beta}\right) / 2 & x \leq R, \\ \left(\left(\frac{2 R-x}{R}\right)^{2 \beta}\right) / 2 & x>R\end{cases}
$$

where $R$ is the distance such that $P(R)=1 / 2$, and $\beta$ is the power attenuation factor ranging between 2 and 6 . We illustrate $P(x)$ in Fig.3(a) by setting $R=40$ and $\beta=4$. One may choose $r=30$ and $\delta=20$ in this example since $P(30) \approx 1.0$ while $P(30+20) \approx 0.0$. Although the actual value of $r$ and $\delta$ may vary for different physical layer models, the key point is that the "gap" $\delta$ is not neglectable compared to the transmission range $r$, which we need to consider in our analysis.

Now we can focus on the determination of the maximum value of $n$ as a function of the successful transmission range $r$ and the channel parameter $\delta$. The results are summarized in the following theorem.

Theorem 1 The number of coding flows (or the maximum encoding number) $n$ in any possible coding structure is upper bounded by $O\left((r / \delta)^{2}\right)$ in $2 D$ space, and $O\left((r / \delta)^{3}\right)$ in $3 D$ space.

Proof: We first consider the 2D case. Based on the above 


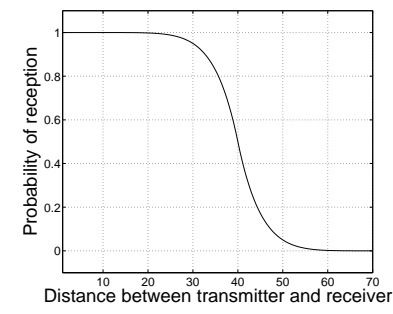

(a) $P(x)$ is a continuous decreasing function of the distance between a transmitter and a receiver.

Fig. 3. Characteristics of transmission range under a typical fading model.

discussion, for each group of $V_{j}^{i}, D_{i}$ and $D_{j}$, we have the distance relationship as shown in Fig. 3(b) (the relationship is a result of the triangular inequality). Obviously, every two receivers $D_{i}$ and $D_{j}$ must be at least $\delta$ apart from each other. Equivalently, each circle with radius $\delta / 2$ centered at a receiver must be disjoint with each other. Meanwhile, each receiver $D_{i}$ must be located within the successful transmission range (denoted by $r$ ) of the coding node $C$. We show such scenario in Fig. 4(a). The question is how many small circles with radius $\delta / 2$ can we pack in a big circle with radius $r$. This number is upper bounded by $O\left((r / \delta)^{2}\right)$, which is also the upper bound for $n$, the maximum encoding number in a coding structure. For 3D case, the circles become spheres and we can carry similar analysis to show the upper bound is $O\left((r / \delta)^{3}\right)$. (a) Packing circles in a circle.

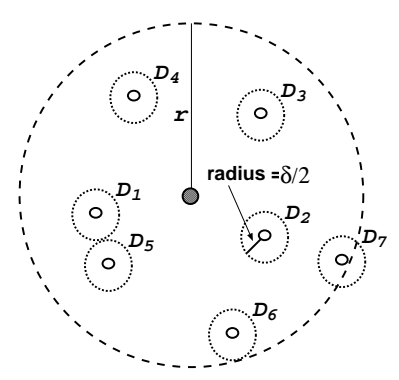

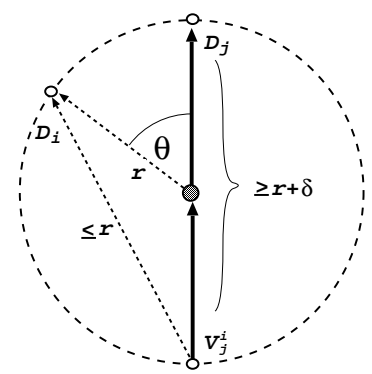

(b) Distance relationship in Fig.2(b).
Fig. 4. Geometrical constraints that bound the number of coding flows $n$.

Remark: The bound in Theorem 1 applies to all possible coding structures under COPE. It does not assume the transmitter for opportunistic listening to be within only one-hop from the coding node. On the other hand, for the representative coding structure shown in Fig.2(b), one can further provide a tighter bound.

Theorem 2 For the coding structure in Fig.2(b), the number of coding flows (or maximum encoding number) is upper bounded by $\pi / \arccos (r /(r+\delta))$.

Proof: For the coding structure in Fig. 2(b), we show the distance relationship in Fig. 4(b). Clearly there is a minimum-
TABLE I

MAXIMUM ENCODING NUMBER: NUMERICAL EXAMPLES.

\begin{tabular}{|c||c|c|c|c|}
\hline$r /(r+\delta)$ & 0.6 & 0.7 & 0.8 & 0.9 \\
\hline \hline max encoding no. & 3.3879 & 3.9497 & 4.8820 & 6.9654 \\
\hline
\end{tabular}

sized arc separating every $D_{i}$ and $D_{j}$, which holds for any two receivers along the circle. Accordingly, the size of the minimum central angle $(\theta$ in Fig. 4(b)) separating any two receivers is $2 \arccos (r /(r+\delta))$, and the maximum number ${ }^{2}$ of coding flows in this structure is $\pi / \arccos (r /(r+\delta))$.

Remark: Table I shows the bound in Theorem 2 for different values of $r /(r+\delta)$. Surprisingly, the maximum number of coding flows (or encoding number) is quite small. This explains why the encoding number observed by the authors of [5] is at most 5 , and in most cases, only 2 to 4 .

\section{Generalization of Coding Structure in Multi-Hop Net- works}

In the above we have limited the coding structure to be within two hops. This is mainly for the simplicity of argument. We can actually extend the coding structure to be more than two hops. One example is shown in Fig. 5, where there are two flows $1 \rightarrow 2 \rightarrow 3 \rightarrow 4$ and $5 \rightarrow 3 \rightarrow 6 \rightarrow 7$ intersecting at node 3 . Node 3 can encode packets from these two flows and broadcast the encoded packets to both node 4 and 6 . Although node 6 cannot perform the necessary overhearing for decoding, it can forward the encoded packet to node 7 , where the opportunistic overhearing and decoding can take place.

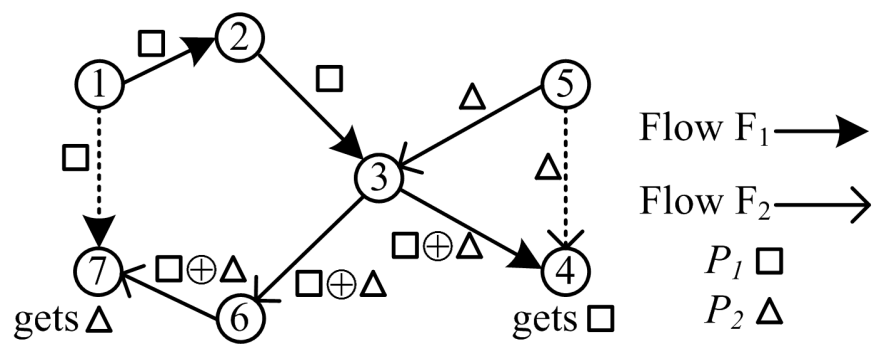

Fig. 5. Generalized coding structure.

In general, both the source node (i.e. Node $S_{i}$ in Fig. 2(a)) and the destination node (i.e. Node $D_{i}$ in Fig. 2(a)) can be multiple hops from the coding node, as long as necessary overhearing has happened before the packets reach the coding node. We now show that Theorem 1 holds for the generalized coding structure as well.

Theorem 3 Theorem 1 holds for generalized coding structure, where node $S_{i}$ and $D_{i}$ in the "logical view" (Fig. 2(a)) can be multiple hops from the coding node $C$.

\footnotetext{
${ }^{2}$ Similarly, this bound can be extended to a 3D case, by changing the "minimum arc" into the "minimum area" on the sphere.
} 
Proof: We can reuse Fig. 3(b) without loss of generality. In this case $V_{j}^{i}, D_{j}$ and $D_{i}$ can be multiple hops away from the coding node, however they must obey the same distance constraint for any valid coding/decoding to happen. Therefore we can get the same results by following the same arguments in Theorem 1.

Remark: It is not hard to imagine that in a multi-hop network, there can be coding opportunities at many relay nodes along the flow, each forming a generalized coding structure with the necessary senders and decoders. Our analysis on the coding structure serves as the building block for analyzing larger network employing distributed and localized network coding.

\section{Coding Performance under Random Access Link-Scheduling}

In previous section, we derived the upper bound of maximum encoding number for any possible coding structures under COPE. Note that the maximum encoding number is achieved by the optimal conditions stated in Lemma 2. Now for a given coding structure with $n$ coding flows, we examine the average encoding number when the link-scheduling uses generic random access mechanism. We will first assume that the coding node never delays transmission, i.e., it competes for channel access whenever it has packets to send and encodes as many packets as possible. At the later part of this section, we will relax this assumption and analyze the performance when a coding node uses delaying strategies.

\section{A. Key Intuition}

Before delving into the analysis, let us first present the high-level intuition that underlies the results in the rest of this section. Consider a coding structure with $n$ coding flows operating under the coding scheme. The number of packets encoded by the coding node is closely related to number of its buffered packets at the instant right before the transmission. If we classify packets in coding node's buffer into $n$ groups, each containing only the packets of one coding flow, then the encoding number is exactly the number of non-empty groups at the instant right before coding node transmits. In other words, higher packet diversity in the buffer will result in higher encoding number.

Two main factors that affect the number of buffered packets at the coding node are traffic volume and random access mechanism. We discuss the effect of traffic volume first. If there is only light traffic across the coding structure, the coding node will have lots of opportunities to transmit its packets before accumulating a large number of non-empty groups in its buffer. On the other hand, if the coding structure is nearly saturated, packets in the coding node's buffer will accumulate and have more non-empty groups. In short, the effect of coding becomes more prominent as the traffic volume increases. We should also emphasize here that the encoding number tends to be larger when the traffic rates of the coding flows are comparable to each other.

The link-level random access mechanism also crucially affects the number of buffered packets. For instance, consider using the basic DCF of 802.11 under heavy traffic. Because the coding node has equal channel access opportunity as all its contenders (i.e., other source nodes), packets may accumulate quickly in its buffer, resulting in a high encoding number. On the other hand, if we try to assign a higher channel access priority to the coding node (since it is most likely the bottleneck node), as suggested by the backward pressure ${ }^{3}$ scheme proposed in [14], then the coding node can clear out the buffered packets faster such that the encoding number will be smaller. We should note that the backward pressure is clearly a good choice for non-coding scheme, because it reduces the self-interference between upstream and downstream nodes along the flow. However, when a coding scheme is employed, one may prefer a higher buffer occupation at the coding node so as to increase the coding opportunity.

\section{B. Calculating the Average Encoding Number}

Based on the above discussion, we use the following stochastic model to capture the dynamics of the coding node's buffer by taking the traffic volume and random access mechanisms into consideration. For simplicity of derivation, we assume a separate buffer structure at the coding node: the coding node maintains a separate buffer for each coding flow. We will show later (in Section V) that the analytical results from separate buffer structure matches well even when one uses a single buffer structure.

Let $M$ denote the buffer size for each coding flow at the coding node. Consider the buffer for one specific coding flow, say flow $S_{i}-C-D_{i}$ (following the notations in Fig. 2(a)). We use an embedded Markov chain to represent the dynamic of this tagged buffer at the coding node and it is illustrated in Fig. 6. The embedded points are right before each successful packet transmission by a source node or the coding node, and we call the interval between two consecutive observation points a slot. Given certain traffic loading at the source nodes, the slot time is a random variable relating to the back-off mechanism and collision probability. For the rest of this paper, the throughput is expressed in the unit of "packet/slot", and the total bandwidth for any random access mechanism is clearly 1 packet/slot.

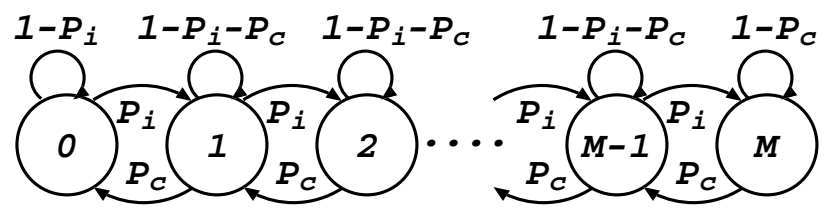

Fig. 6. Embedded Markov chain for the tagged buffer.

\footnotetext{
${ }^{3}$ Whenever the downstream node has packets to send, it will restrain the transmission of upstream node.
} 
Now we consider the state transitions of this embedded Markov chain. Let $P_{i}(i=1, \ldots, n)$ denote the probability that the source node $S_{i}$ transmits in the corresponding slot, and let $P_{c}$ denote the probability that the coding node transmits in the corresponding slot. Let $N_{i}(t)$ be the random variable representing the number of buffered packets at the coding node for the $i$-th flow at slot $t$, then we have the following events at each state transition:

- If $S_{i}$ transmits, then $N_{i}(t+1)=N_{i}(t)+1$ if $N_{i}(t)<M$, and $N_{i}(t+1)=N_{i}(t)=M$ otherwise.

- If the coding node transmits, then $N_{i}(t+1)=N_{i}(t)-1$ if $N_{i}(t)>0$, and $N_{i}(t+1)=N_{i}(t)=0$ otherwise.

- If $S_{j}$ (for $j \neq i$ ) transmits, then $N_{i}(t+1)=N_{i}(t)$.

Let $\pi_{j}^{i}$ denote the steady state probability that the tagged buffer (for flow $i$ ) has $j$ packets. Solving this embedded Markov chain, we have

$$
\pi_{j}^{i}=\left(\alpha_{i}\right)^{j}\left[\frac{1-\alpha_{i}}{1-\left(\alpha_{i}\right)^{M+1}}\right], \quad j=0,1, \ldots, M
$$

where $\alpha_{i}=P_{i} / P_{c}$. The probability that the tagged buffer is not empty, denoted as $\kappa_{i}$, is

$$
\kappa_{i}=1-\pi_{0}^{i}=\frac{\alpha_{i}-\left(\alpha_{i}\right)^{M+1}}{1-\left(\alpha_{i}\right)^{M+1}} .
$$

Define $\Omega_{i}$ as a random variable such that $\Omega_{i}=1$ if the tagged buffer is not empty, and $\Omega_{i}=0$ otherwise. Then the average encoding number per slot can be expressed as

$$
E[\Omega]=\sum_{i=1}^{n} E\left[\Omega_{i}\right]=\sum_{i=1}^{n} \kappa_{i} .
$$

We can express the total effective throughput, denoted by $T$, as

$$
T=E[\Omega] P_{c}
$$

and the effective throughput for the $i^{\text {th }}$ coding flow is simply $T_{i}=\kappa_{i} P_{c}$.

The remaining issues are to derive the transmission probabilities $P_{i}$ and $P_{c}$, which are determined by the traffic volume and random access mechanism. We use the "contending probability", $\rho_{i}$ or $\rho_{c}$, to denote the probability that node $S_{i}$ or node $C$ competes for channel access at each slot. We have the following expression for $\rho_{c}$ :

$$
\rho_{c}=1-\prod_{i=1}^{n}\left(1-\kappa_{i}\right) .
$$

Now we model the effect of random access. We are particularly interested in two generic classes of random access mechanisms: 1) equal access (i.e., all transmitters having the same priority for channel access), and 2) higher priority for the relay (coding) node (e.g., backward pressure in [14]).

Equal Access: When all competing nodes have equal channel access probability, then the probability that node $S_{i}$ transmits in a slot, conditioned that it is the contender for this slot, is $1 /\left(\rho_{c}+\sum_{j \neq i} \rho_{j}+1\right)$, and the probability that the coding node transmits conditioned that it contends is $1 /\left(1+\sum_{i} \rho_{i}\right)$. We can express $P_{i}$ and $P_{c}$ as:

$$
P_{i}=\rho_{i} \frac{1}{\rho_{c}+\sum_{j \neq i} \rho_{j}+1}, \quad P_{c}=\rho_{c} \frac{1}{1+\sum_{i} \rho_{i}} .
$$

K-Priority: Assume the relay (coding) node has " $K$-priority" over its upstream nodes, namely, the coding node gets $K$ times $(K>1)$ of the opportunity that another competing source node transmits. Then $P_{i}$ and $P_{c}$ can be expressed as:

$$
P_{i}=\rho_{i} \frac{1}{K \rho_{c}+\sum_{j \neq i} \rho_{j}+1}, \quad P_{c}=\rho_{c} \frac{K}{K+\sum_{i} \rho_{i}} .
$$

\section{Case Studies}

We conduct several case studies to gain the important insights on the effect of traffic volume and random access.

Case 1-Saturation Throughput: We first examine the saturation throughput of both "equal access" and "K-priority" mechanisms. By saturation, we mean that each source node always has backlogged packets to transmit. Under such condition, we have $\rho_{i}=1$ for all $i=1, \ldots, n$. Combining Eq. $(2)(5)(6)(7)$, we have the following fixed-point equation:

$$
\rho_{c}=1-\left(\frac{1-\alpha}{1-\alpha^{M+1}}\right)^{n}
$$

where

$$
\alpha= \begin{cases}\frac{1+n}{\rho_{c}\left(\rho_{c}+n\right)} & \text { (for Equal Access) } \\ \frac{K+n}{K \rho_{c}\left(K \rho_{c}+n\right)} & \text { (for } K \text {-priority) }\end{cases}
$$

Given $n, K$ and $M$, one can find $\rho_{c}$ using numerical method, and then calculate the average encoding number and throughput using Eq. (3)(4). In particular, we find that for the "equal access" mechanism, $\rho_{c}=1$ is a good enough approximation for all $n>1$ and $M \geq 1$ cases. Therefore, the average encoding number of equal access under saturation is

$$
E[\Omega]=n \frac{M}{M+1},
$$

and the corresponding total throughput is

$$
T=E[\Omega] P_{c}=\left(\frac{n}{n+1}\right)\left(\frac{M}{M+1}\right) .
$$

Nonetheless, for the " $K$-priority" mechanism, $\rho_{c}=1$ is no longer a good approximation especially when $K$ is relatively large. The main reason behind is that the coding node now clears its buffer faster such that it is less likely to have packets for transmission.

For instance, we set $n=4$ (i.e., 4 coding flows), and find $\rho_{c}$ (and hence average encoding number and throughput) for different values of buffer size $(M)$ and priority $(K)$ using numerical method. We illustrate the interplay of encoding number, throughput and buffer for different values of $K$ in Fig. 7, which shows that higher priority $(K)$ results in both lower encoding number and lower throughput in most cases. In particular, when the coding node has a very high priority (e.g., 
$K=10$ ), nearly all the coding opportunities are diminished such that the throughput of coding scheme is only around 0.5 , the optimal throughput of the non-coding scheme.

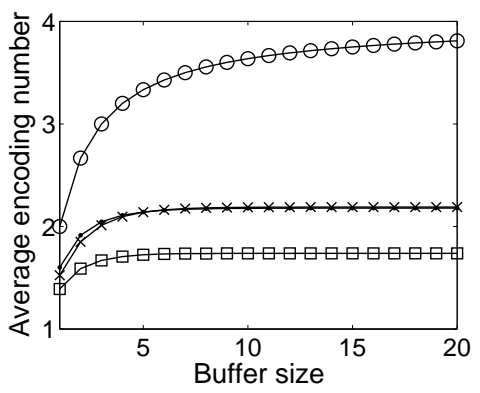

(a) Average encoding number.

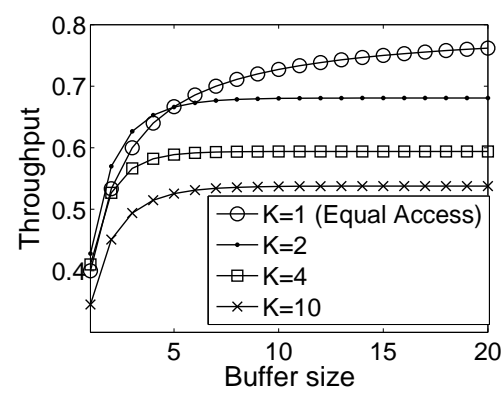

(b) Total throughput.

Fig. 7. Interplay of buffer size $(M)$, encoding number, $K$-priority and throughput under saturation.

Remark: We have observed the advantage of "equal access" over "K-priority" especially with relatively large buffer size (i.e., $M>5$ ). Recall from Lemma 2 that the optimal throughput for the coding scheme is $n B /(n+1)$ with $B=1$ packet/slot here. Now we can see that the equal random access adds a fraction of $M /(M+1)$ onto the optimal encoding number as well as the throughput! A large buffer size (say $M>10$ ) can alleviate the performance degradation, but will induce longer queuing time at the coding node and hence longer delay. This illustrates an important tradeoff between throughput and delay at the coding node.

Case 2-Feasible and Optimal Bandwidth Allocation: The transmission probabilities $P_{i}, P_{c}$ essentially reflect the bandwidth share among the source nodes $S_{i}(i=1, \ldots, n)$ and the coding node. Based on Eq. (6) and (7), we can examine the feasible bandwidth allocation of both "equal access" and " $K$ - priority" as follows: given a desired set of bandwidth allocation $\left\{P_{i}\right\} \cup P_{c}$, we can put the values of $P_{i}$ and $P_{c}$ into Eq. (6) or (7) and get $\left\{\rho_{i}\right\} \bigcup \rho_{c}$. The desired bandwidth allocation is feasible only if $0 \leq \rho_{i} \leq 1 \forall i$ and $0 \leq \rho_{c} \leq 1$.

We formulate the optimal bandwidth allocation problem as follows: given a desired proportional bandwidth allocation among the source nodes, i.e., $\gamma_{1}, \ldots, \gamma_{n}$ such that $P_{1}: P_{2}$ : $\cdots: P_{n}=\gamma_{1}: \gamma_{2}: \cdots: \gamma_{n}$, determine the value of $P_{c}$ so as to maximize the total end-to-end throughput. To solve the
TABLE II

OPTIMAL VALUES OF “ $K$ ” WITH VARYING BUFFER SIZE.

\begin{tabular}{|c||c|c|c|c|c|}
\hline$M$ & 2 & 5 & 7 & 10 & 20 \\
\hline \hline$P_{c}^{*}$ & 0.296 & 0.251 & 0.239 & 0.229 & 0.215 \\
\hline$T^{*}$ & 0.576 & 0.696 & 0.723 & 0.745 & 0.772 \\
\hline avg. encoding \# & 1.95 & 2.77 & 3.03 & 3.26 & 3.58 \\
\hline$K^{*}$ & 1.32 & 1.19 & 1.15 & 1.11 & 1.06 \\
\hline
\end{tabular}

problem, we can express $\alpha_{i}$ as

$$
\alpha_{i}=\frac{P_{i}}{P_{c}}=\frac{\gamma_{i}\left(1-P_{c}\right) / \sum_{i} \gamma_{i}}{P_{c}} \quad i=1, \ldots, n,
$$

and combine with Eq. (2)(3)(4) to express the total throughput as a function of $P_{c}$. In particular, given the number of coding flows $n$ and the buffer size $M$, one can show that the maximum total end-to-end throughput is achieved when all source nodes get equal bandwidth share, i.e., $\gamma_{1}=\cdots=\gamma_{n}$.

For instance, when $n=4, \gamma_{1}=\gamma_{2}=\gamma_{3}=\gamma_{4}$, we obtain the optimal $P_{c}^{*}$ and throughput $T^{*}$ for different values of buffer size $M$. Furthermore, we obtain the optimal values of $K$ such that the corresponding optimal bandwidth allocation is feasible for the random access mechanisms. The results are summarized in Table II. One can observe that with higher buffer size $(M)$, the optimal bandwidth share of the coding node is lower while both the throughput and average encoding number is higher. The "equal access" (i.e., $K=1$ ) tends to be closer to the optimal with larger buffer size.

Case 3-Performance under Adequate Buffer Size: We have seen that a large buffer size is essential in utilizing the coding opportunities, and it is with large buffer size that "equal access" outperforms " $K$-priority". To further explore the reason behind, let us assume adequate buffer size (say $M \geq 20$ ), and consider a general case where the coding flows may have asymmetric traffic rates, i.e., $P_{1}, P_{2}, \ldots, P_{n}$ may not be equal to each other.

Let $L_{i}$ denote the packet loss ratio for flow $i$ at the coding node. From Eq. (1), one can show that the packet loss ratio for flow $i$ at the coding node is equal to $\pi_{M}^{i}$.

$$
L_{i}=\pi_{M}^{i}=\left(\alpha_{i}\right)^{M}\left[\frac{1-\alpha_{i}}{1-\left(\alpha_{i}\right)^{M}+1}\right]
$$

Let's say $\alpha_{i} \leq 1$ (i.e. $P_{c} \geq P_{i}$ ), then

$$
L_{i} \leq\left.\left(\alpha_{i}\right)^{M}\left[\frac{1-\alpha_{i}}{1-\left(\alpha_{i}\right)^{M}+1}\right]\right|_{\alpha_{i} \rightarrow 1}=\frac{1}{M+1}
$$

which is negligible when $M$ is large enough. In other words, the prerequisite for low packet loss for any coding flow is only $P_{c} \geq P_{i} \forall i$.

Now we let $P_{c}=\max _{i}\left\{P_{i}\right\}$ and examine whether such bandwidth allocation is feasible for "equal access" or " $K$ priority". From Eq. (6) and (7), we can see that such allocation is feasible for "equal access" by simply letting $\rho_{c}=\max _{i}\left\{\rho_{i}\right\}$, while " $K$-priority" fails to provide a feasible solution even with small values of $K$ (e.g., $K=2)$. This 
explains why "random access" is particularly suitable for the coding scheme with adequate buffer size.

\section{$D$. Will Delaying Strategy at the Coding Node Help?}

In the above analysis, we assume the coding node competes for channel access whenever it has packets in the buffer. Now we discuss the delaying strategies of the coding node. We call such strategies as "Wait-for- $X$ ", namely, letting the coding node hold transmission until it can encode at least $X$ packets. By such scheme, the average encoding number is at least $X$. Although it seems to be promising at the first glance, it has the following drawbacks.

First of all, the effect of "Wait-for- $X$ " on increasing the encoding number is only significant when $X$ is large enough (e.g., $X \geq M /(M+1)$ with symmetric flow rates), and in many cases, only when $X=n$. However, a large $X$ means the coding node may hold back its transmission for a longer time, which will significantly increase the packet loss ratio, unless the coding node also has a very high " $K$-priority".

Secondly, "Wait-for- $X$ " increases encoding number significantly only when the network is far from saturation and the buffer size is relatively small. However, trying to have a high encoding number when there is only light traffic load has little benefit on the throughput because the network can sustain the traffic even without network coding at all. When the traffic intensity increases, the encoding number can "automatically" increase due to higher buffer occupancy. We have shown that when the network is operating close to saturation, the "equal access" with a moderate buffer size at the coding node is sufficient to utilize most of the coding opportunity.

Last but not least, when the coding flows have asymmetric traffic rates, "Wait-for- $X$ " will easily lead to buffer overflow because it takes more time to accumulate enough packets for encoding compared to the symmetric case.

\section{Fundamental Limits of the Coding Scheme}

In previous two sections, we have characterized the basic coding structure and examined its performance under various random access mechanisms. Now we provide an upper bound on the throughput gain for a general wireless network, i.e., a network with any possible topology and traffic demand. In such setting, there are two main differences from the single coding structure case: 1 ) there may exist several coding structures in the network; 2) there may exist "non-coding flows", i.e., flows that are not relevant to any coding process, and these non-coding flows may even interfere with the coding flows.

Let us first define the throughput for a general network. Given a set of traffic demands $\left\{D_{i}\right\}$, each containing a source node, a destination node, and a traffic value denoted as $\lambda_{i}$, the throughput scale-up of the network is a positive real number $k$ such that the set of flow rates $\left\{k \lambda_{i}\right\}$ can be supported by the network. We use $k_{c}^{*}$ and $k_{n c}^{*}$ to denote the maximum throughput scale-up achieved by the coding and non-coding schemes respectively. The throughput gain, denoted as $G$, is equal to $k_{c}^{*} / k_{n c}^{*}$, and the maximum throughput gain, denoted as $G^{*}$, is the maximum value of $G$ over all possible network topologies, traffic demands, link-scheduling and routing algorithms.

One should note that the coding scheme and non-coding scheme may use different routes to achieve their respective maximum throughput. However, the following lemma states that to obtain the upper bound of $G^{*}$, we only need to consider a smaller feasible space.

Lemma 3 For a general wireless network, the maximum throughput gain $G^{*}$ is upper bounded by the maximum throughput gain when both coding and non-coding schemes operate under the same routes, over all feasible routing policies.

Proof: Given the network topology and the set of traffic demands, we use $R$ to denote set of routes chosen for the flows. We use $k_{c}^{*}(R) \quad\left(k_{n c}^{*}(R)\right)$ to denote the maximum throughput scale-up when using the routes $R$ for coding (noncoding) scheme. Let $R_{c}^{*}$ and $R_{n c}^{*}$ denote the optimal routes for coding and non-coding schemes respectively. Then we have

$$
\mathcal{G}^{*}=\frac{k_{c}^{*}}{k_{n c}^{*}}=\frac{k_{c}^{*}\left(R_{c}^{*}\right)}{k_{n c}^{*}\left(R_{n c}^{*}\right)} \leq \frac{k_{c}^{*}\left(R_{c}^{*}\right)}{k_{n c}^{*}\left(R_{c}^{*}\right)}
$$

which says that the general maximum throughput gain is upper bounded by the maximum throughput gain conditioned on the same routing for both coding and non-coding schemes. Therefore, to examine the maximum throughput gain, we can only consider the case when both schemes choose the same routes for all flows, such that the same coding structures exist in both schemes.

Now we consider the impact to a single coding structure when there is interference from other non-coding flows. One important observation is that non-coding flows that interfere with the coding node can also benefit from the coding scheme, because the coding node can send out the same amount of information by consuming less bandwidth, thus leaving more bandwidth for other competing nodes, including the non-coding flows. In case that there exists non-coding flows sharing the bandwidth with coding flows, the following lemma provides an upper bound on throughput gain by the coding scheme within a single coding structure.

Lemma 4 For a single coding structure with possibly noncoding flows interfering with the coding node, the maximum throughput gain for both the coding flows and non-coding flows is upper bounded by $2 n /(n+1)$ when the buffer size $M$ at the coding node approaches infinity, and this upper bound can be approximated as $2 n /\left(n+\frac{M}{M+1}\right)$.

Proof: Fig. 8 illustrates the scenario that a coding structure may encounter in a general wireless network. The coding flows can be longer than two hops, and there can be non-coding 
flows (e.g., flow $1 \rightarrow 2 \rightarrow 3, C \rightarrow 4$ and $5 \rightarrow 6$ in Fig. 8) interfering with the coding node $C$.

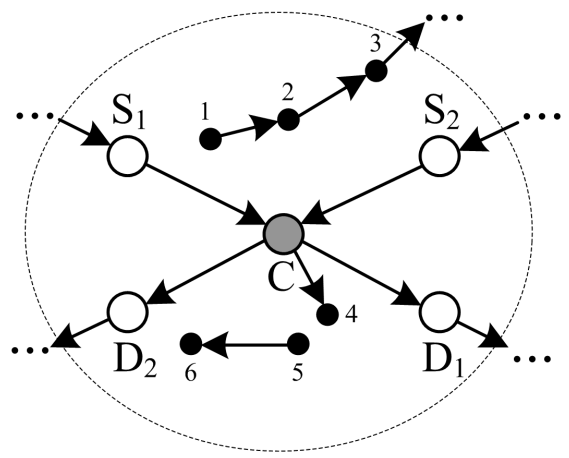

Fig. 8. Illustration of the coding structure within a general wireless network.

For the coding scheme, we use $\lambda_{i}^{c}(i=1, \ldots, n)$ to denote the throughput of coding flow $i$, and use $\lambda_{o}^{c}$ to denote the total throughput of all non-coding flows that interfere with the coding node. In addition, we use $\lambda_{c}^{c}$ to denote the bandwidth consumed by the coding node $C$ for all the coding flows.

Similarly, for the non-coding scheme, we use $\lambda_{i}^{n c}(i=$ $1, \ldots, n)$ to denote the throughput of the flow that travels through $S_{i}-C-D_{i}$, and use $\lambda_{o}^{n c}$ to denote the total throughput of all the other flows that interfere with node $C$. In addition, we use $\lambda_{c}^{n c}$ to denote the bandwidth consumed by node $C$ for flows $S_{i}-C-D_{i}(i=1, \ldots, n)$.

Now we observe the bandwidth consumption from the perspective of node $C$. For the coding scheme, we use $B_{c}$ to denote the total bandwidth consumed by node $C$ and all its competing nodes. Clearly $B_{c}$ is a function of $\lambda_{i}^{c}(i=1, \ldots, n)$, $\lambda_{c}^{c}$ and $\lambda_{o}^{c}$. For instance, in Fig. 8, flow $1-2-3$ has two nodes interfering with node $C$, so the amount of bandwidth it contributes in $B_{c}$ is equal to two times of its throughput. Generally, we can express $B_{c}$ as

$$
B_{c}=\sum_{i=1}^{n} \nu_{i} \lambda_{i}^{c}+\lambda_{c}^{c}+\nu_{o} \lambda_{o}^{c}
$$

where $\nu_{i} \geq 1$ for all $i$ and $\nu_{o} \geq 1$.

Similarly, for the non-coding scheme, we have

$$
B_{n c}=\sum_{i=1}^{n} \nu_{i} \lambda_{i}^{n c}+\lambda_{c}^{n c}+\nu_{o} \lambda_{o}^{n c}
$$

The following observation relates $B_{c}$ and $B_{n c}$ :

Observation 1: We have $B_{c} \leq B_{n c}$, when both coding and non-coding schemes are maximizing their throughput (based on the same routing). We can prove this observation by contradiction: if $B_{c}>B_{n c}$, then there is clearly some "free" bandwidth (which can increase throughput) that is not utilized by the non-coding scheme. However, we assume that the noncoding scheme is maximizing the throughput. This shows the contradiction.
Recall the results in Section III and Lemma 1, we have the following observation:

Observation 2: When both coding and non-coding scheme are maximizing their throughput, we have: 1) $\lambda_{c}^{c}=$ $\max _{i}\left\{\lambda_{i}^{c}\right\}$ with infinite buffer size, and approximately $\lambda_{c}^{c}=$ $\frac{M+1}{M} \max _{i}\left\{\lambda_{i}^{c}\right\}$ with adequate buffer size; 2) $\lambda_{c}^{n c}=$ $\sum_{i=1}^{n} \lambda_{i}^{n c}$.

With infinite buffer size, combining Eq. (15)(16) and the above observations, we have

$$
\sum_{i=1}^{n} \mathcal{G} \nu_{i} \lambda_{i}^{n c}+\mathcal{G} \max _{i}\left\{\lambda_{i}^{n c}\right\}+\mathcal{G} \nu_{o} \lambda_{o}^{n c} \leq B_{n c}
$$

Therefore, we have

$$
\begin{aligned}
\mathcal{G} & \leq \frac{B_{n c}}{\sum_{i=1}^{n} \nu_{i} \lambda_{i}^{n c}+\max _{i}\left\{\lambda_{i}^{n c}\right\}+\nu_{o} \lambda_{o}^{n c}} \\
& =\frac{B_{n c}}{B_{n c}-\lambda_{c}^{n c}+\max _{i}\left\{\lambda_{i}^{n c}\right\}} \\
& =\frac{B_{n c}}{B_{n c}-\sum_{i=1}^{n} \lambda_{i}^{n c}+\max _{i}\left\{\lambda_{i}^{n c}\right\}} \\
& \leq \frac{B_{n c}}{B_{n c}-\sum_{i=1}^{n} \lambda_{i}^{n c}+\frac{1}{n} \sum_{i=1}^{n} \lambda_{i}^{n c}} \\
& \leq \frac{B_{n c}}{B_{n c}-\frac{n-1}{n} \frac{B_{n c}}{2}} \\
& =\frac{2 n}{n+1}
\end{aligned}
$$

With adequate buffer size, we have

$$
\begin{aligned}
\mathcal{G} & \leq \frac{B_{n c}}{\sum_{i=1}^{n} \nu_{i} \lambda_{i}^{n c}+\frac{M+1}{M} \max _{i}\left\{\lambda_{i}^{n c}\right\}+\nu_{o} \lambda_{o}^{n c}} \\
& =\frac{B_{n c}}{B_{n c}-\lambda_{c}^{n c}+\frac{M+1}{M} \max _{i}\left\{\lambda_{i}^{n c}\right\}} \\
& =\frac{B_{n c}}{B_{n c}-\sum_{i=1}^{n} \lambda_{i}^{n c}+\frac{M+1}{M} \max _{i}\left\{\lambda_{i}^{n c}\right\}} \\
& \leq \frac{B_{n c}}{B_{n c}-\sum_{i=1}^{n} \lambda_{i}^{n c}+\frac{M+1}{M} \frac{1}{n} \sum_{i=1}^{n} \lambda_{i}^{n c}} \\
& \leq \frac{B_{n c}}{B_{n c}-\left(1-\frac{M+1}{n M}\right) \frac{B_{n c}}{2}} \\
& =\frac{2 n}{n+\frac{M+1}{M}}
\end{aligned}
$$

Another key insight that enables us to get the upper bound for a general wireless network is that the coding scheme only brings local improvement. In particular, coding scheme only increases the bandwidth efficiency of the coding node. Consider the two cases shown in Fig. 9, where there are non-coding flows interfering with either $S_{1}, S_{2}$ (Fig. 9(a)) or the coding node $C$ (Fig. 9(b)). In such cases, there is not much room left for the coding flows to improve their throughput, and the overall throughput gain is diminished. Moreover, if a coding flow traverses several coding structures, its end-to-end throughput is upper bounded by the bottleneck 
coding structure: its throughput improvement is bounded by the throughput gain in one of the traversed coding structures which gives it the least improvement. Therefore, we have the following result.

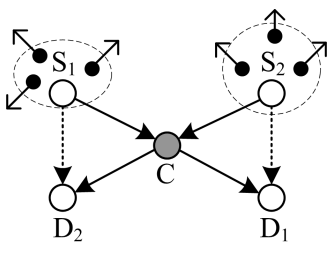

(a) Excessive contention at node $S_{1}, S_{2}$

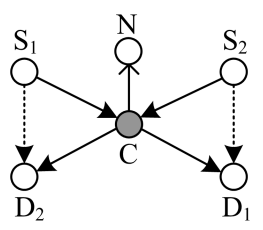

(b) Non-coding flow interferes with $C$.
Fig. 9. Two scenarios that limit the throughput gain by coding.

Theorem 4 For a general wireless network, the end-to-end throughput gain $G$ by using the XOR coding is upper bounded by $2 n /(n+1)$ when the buffer size $M$ at the coding node approaches infinity, and this bound can be approximated as $2 n /\left(n+\frac{M}{M+1}\right)$, where $n$ is the maximum encoding number in one of its coding structures.

Proof: We have shown that each coding structure in the network, say coding structure $k$, can provide a maximum throughput gain $2 n_{k} /\left(n_{k}+1\right)$. Because we ensure the same throughput gain by all flows, the maximum throughput gain is limited by one of the coding structures that provides the least throughput gain. Therefore, this theorem holds.

Remark: 1) Compared to the bound provided in [10], our bound is tighter and holds for any possible topologies. 2) Our definition of throughput gain provides an analytical justification for the "coding gain" defined in [5]. Note that authors of [5] also define a "coding+MAC gain" that can even approach $n$. However, the "coding+MAC gain" compares the saturation throughput of both coding and non-coding schemes, which is not a "fair" comparison because the saturation throughput of non-coding scheme is much lower than the maximum throughput it can achieve.

\section{Verification of the Analysis}

\section{A. Simulation Results in a Single Coding Structure}

We implement a discrete-event simulator that approximates the random access mechanisms including Equal Access, " $K$ Priority" and "Wait-for- $X$ " discussed in Section III. We conduct the simulation in single coding structure, to verify the correctness and precision of the methodology used in this section.

For the coding scheme, we use two types of buffer structure: separate structure (i.e., independently maintaining a buffer for each coding flow) and single structure (i.e., maintaining one large buffer for all coding flows) at the coding node $C$. For the single buffer structure, the buffer size is equal to $n M$, where $n$ is the number of coding flows, and $M$ is the per flow buffer size. We present the simulation results in two main perspectives: 1) performance with fixed traffic load and varying buffer size; 2) performance with adequate buffer size $(M=20)$ and varying traffic load. We normalize the channel bandwidth $B=1$ and the main parameters are $n$ (number of coding flows), $M$ (buffer size per flow), values of $K$ ( $K$ Priority) and $X$ (Wait-for- $X$ ). Note that, although our results hold for any given $n$ in general, we choose $n=4$ in the following experiments for consistency of presentation.

Experiment 1 (Performance of "Equal Access"): Fig. 10 shows the performance with $n=4$ coding flows under saturated condition. Each flow has a traffic load equal to the total bandwidth to saturate the system, and we vary the per flow buffer size at the coding node between 1 and 20 . We depict the results by both the separate buffer structure and the single buffer structure, and compare them with the analytical results. By the analysis, the saturated throughput and packet loss ratio can be approximated as $\frac{n}{n+1} \frac{M}{M+1}$ and $\frac{1}{M+1}$ respectively. One can see that the analytical results match very closely to the simulation results. Note that for the non-coding scheme, the throughput and packet loss are not sensitive to buffer size.

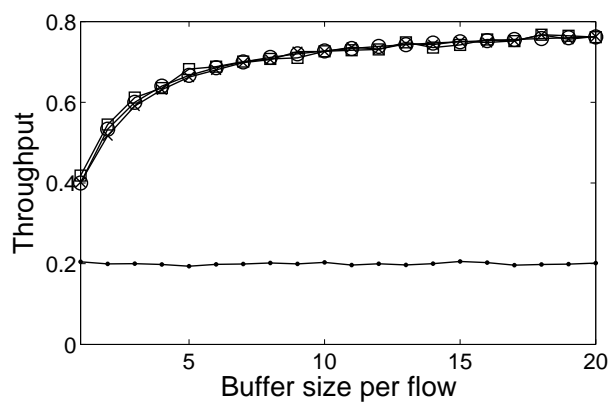

(a) Total throughput.

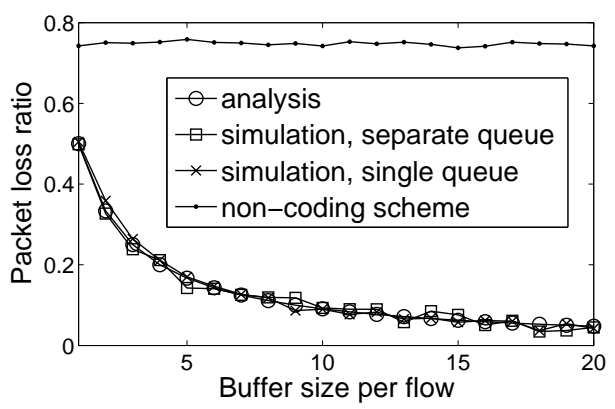

(b) Packet loss ratio.

Fig. 10. Results with $n=4$ coding flows under saturation and equal access.

To further observe the performance with adequate buffer size, we set the per flow buffer size $(M)$ to 20 and vary traffic load of the source nodes. In Fig. 11(a), we keep increasing the symmetric offered load and observe the total throughput $T$. Based on the analysis, the optimal bandwidth allocation for achieving maximum throughput is $P_{c}^{*} \approx 0.22, P_{i}^{*} \approx 0.19$ and the optimal throughput is about 0.77 . One can see that throughput of the coding scheme does approach maximum when the traffic load approaches 0.19 , and the maximum throughput by 
equal access is very close to the optimal. Furthermore, the throughput remains stable as the load increases. Nonetheless, for the non-coding scheme, the saturation throughput (about 0.2 ) is much lower than the maximum throughput (about 0.48) that the system can achieve.

In Fig. 11(b), we illustrate the total throughput with asymmetric flow rates. We fix the traffic load of three existing flows to be $0.05,0.1$ and 0.15 , and keep increasing the load of an incoming flow. We depict both the total throughput and the throughput for the incoming flow. Not surprisingly, the total throughput remains stable at around 0.65 when the arrival rate of the incoming flow exceeds 0.33 . One can check that the optimal bandwidth share for the incoming flow is indeed around 0.33 while the optimal total throughput is around 0.65 . This justifies that equal access with adequate buffer size does lead to close-to-optimal throughput.

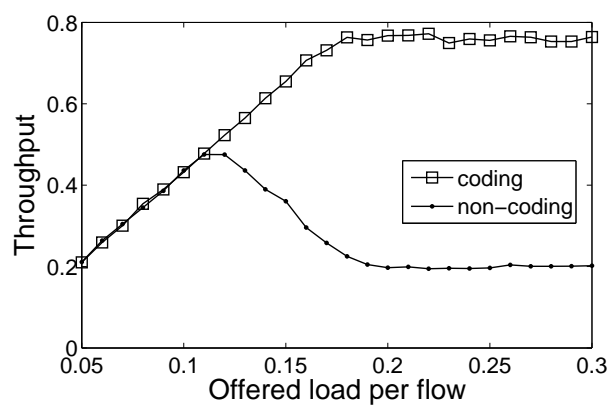

(a) Symmetric flow rate.

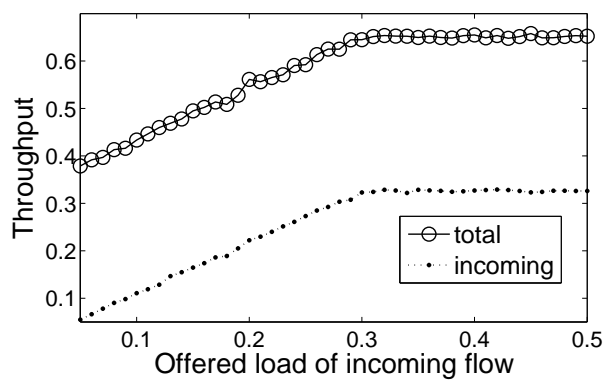

(b) Asymmetric flow rate $(0.05+0.1+0.15+x)$.

Fig. 11. Throughput with $n=4$ and $M=20$ under equal access.

Experiment 2 (Performance of "K-priority" and "Waitfor-X"): Let us first study the performance of the " $K$-priority" mechanism. We set $n=4$ coding flows, each with the offered load of 0.2 , the theoretically optimal bandwidth share stated by Lemma 2 . We compare the total throughput and packet loss ratio of different values of $K(K=1$ is simply the equal access). One can see that the simulation results in Fig. 12(a) matches well with the analytical results shown in Fig. 7(b). The throughput performance of random access outperforms all other $K$-priority schemes when the buffer size $M$ is greater than 5 . Nonetheless, $K$-priority indeed guarantees low packet loss at the relay (coding) node.

In Fig. 13(a), we compare the total throughput of several combinations of " $K$-priority" and "Wait-for- $X$ " schemes. The

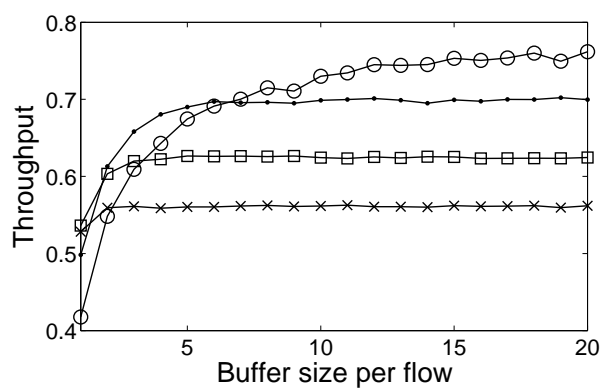

(a) Throughput.

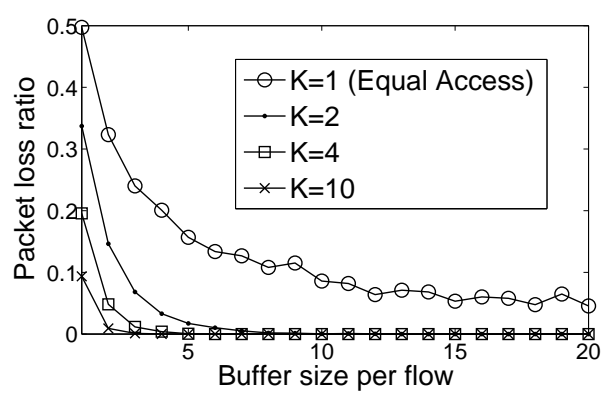

(b) Packet loss ratio.

Fig. 12. Performance of " $K$-priority" mechanism.

offered load of each flow is set to 0.2. As we have discussed, a large value of $X$ with $K=1$ results in the lowest throughput and the highest loss ratio. Even when the "Wait-for- $X$ " scheme is accompanied with a proper " $K$-priority" $(K=10, X=4)$, there is no significant performance improvement compared to the simple $(X=1, K=1)$ pair.

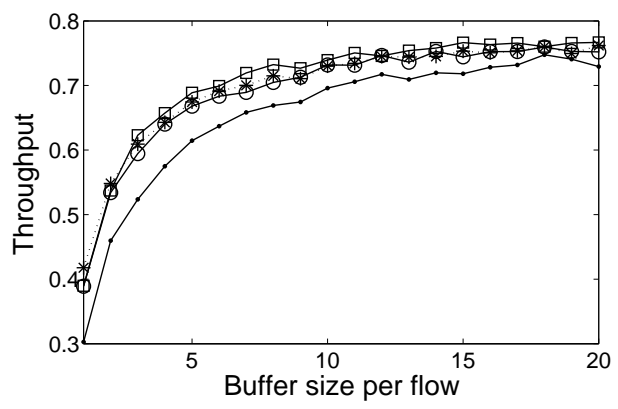

(a) Throughput.

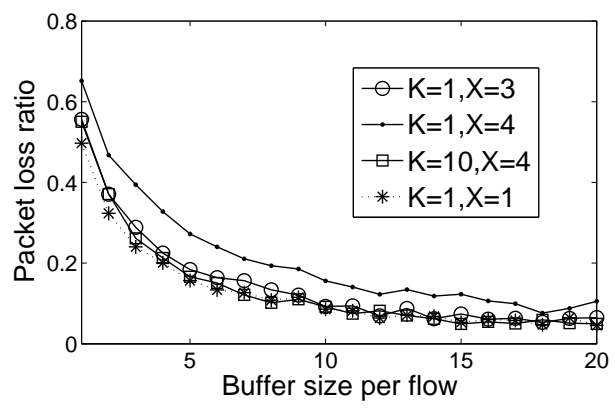

(b) Packet loss ratio.

Fig. 13. Performance of combinations of " $K$-priority" and "Wait-for- $X$ ". 


\section{$B$. Simulation Results under 802.11 and General Networks}

While our analysis is not based on the exact 802.11 DCF, we expect our results to serve as a general guideline for codingrelated protocol design in wireless networks, where 802.11 DCF is the predominant link-scheduling mechanism. In the following experiment, we implement the COPE functionalities under NS-2, and observe the throughput gain in both single coding structure and general wireless networks. Based on our former analysis, we set the per-flow buffer size to be larger than 20 .

Experiment 3 (Throughput gain under 802.11 in a single coding structure): We form a coding structure with $n=2$ coding flows and symmetric flow rates. In Fig. 14(a), we plot the throughput gain with increasing offered load. While the theoretical optimal throughput gain equals to $2 n /(n+1) \approx$ 1.33 , we can see that 802.11 achieves an approximately 1.22 throughput gain due to collisions.

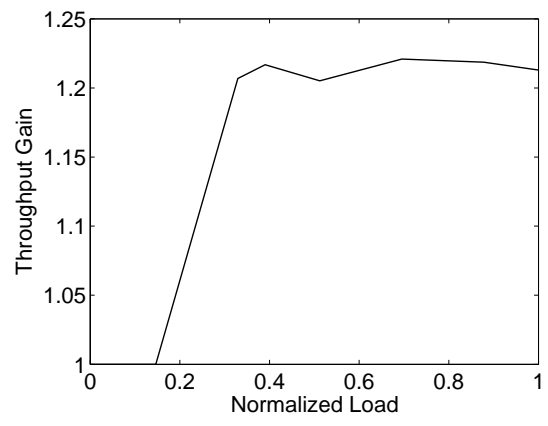

(a) Results in a $n=2$ coding structure.

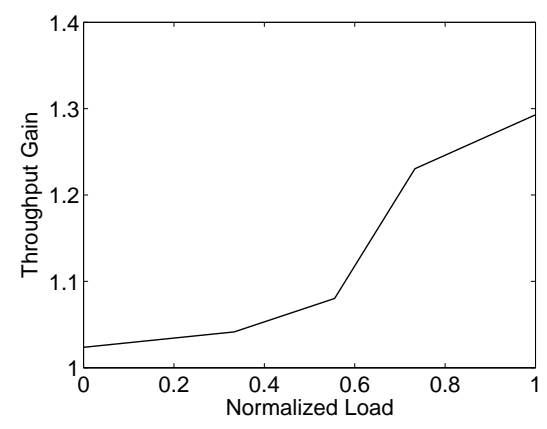

(b) Results in a 4 by 4 grid.

Fig. 14. Throughput gains vs. offered load under NS-2.

Experiment 4 (Throughput gain under 802.11 in a grid topology): We form a 4 by 4 grid topology and the experiment is of 10 rounds. In each round, we randomly add 5 flows into the network and repeat the process for 3 times. We plot the aggregate throughput gain achieved by the coding scheme in Fig. 14(b). The objective of this experiment is to testify the soundness of our bound on the throughput gain proposed in Section IV. To mimic the "optimal routing" for non-coding and coding schemes respectively, we use ETX [4] routing for the non-coding scheme, and our own proposed coding-aware routing [6] for the coding scheme. The end result is that the throughput gain for this multi-hop network, as we discussed in Section IV, is at most 1.3.

\section{Potential Applications}

We now briefly introduce the potential research directions where our analysis can be useful:

1) Designing coding-efficient link-schedulers: A codingefficient link-scheduler should utilize most of the coding opportunities to improve throughput. In Section III, we examine a general class of random access mechanisms that can be used in a coding structure. In particular, we have found the advantage of equal access mechanisms and characterized the importance of buffer size. These insights can be particularly valuable for designing coding-efficient link-schedulers.

2) Deriving per-flow throughput under coding scheme: The effective sending rate of the coding node is simply the product of average encoding number and the physical bandwidth it consumes. In general, the throughput in unit of "packet per second" can be derived as long as the average slot time is calculated based on the random access mechanism used.

3) Designing algorithms for coding-aware routing: In [11], [12], the authors proposed the coding-aware routing. The main reason for incorporating coding considerations into routing algorithm is that the coding opportunity are crucially dependent on traffic pattern, which in turns is affected by the routing decision. If using throughput capacity as the metric for evaluating a new path, our throughput analysis in the coding structure can be useful for determining the throughput capacity of a new path with potential coding opportunity.

\section{Related Works}

The concept of network coding is first proposed in [1]. Since then, the potential benefit of network coding has been studied in various settings. For the wired case, [9] provides various bounds on the throughput gain for single multicast, single/multiple unicast and single broadcast cases. More recently, for multiple unicast sessions in wireless networks, [10] shows that the throughput gain is upper bounded by $\frac{1+\Delta}{1+\Delta / 2}$ in $1 \mathrm{D}$ random networks, and upper bounded by $2 c \sqrt{\pi} \frac{1+\Delta}{\Delta}$ in 2D random networks, where $\Delta$ is a parameter characterizing the intensity of interference, and $c=\max \left\{2, \sqrt{\Delta^{2}+2 \Delta}\right\}$. It is conjectured in [10] that the throughput gain is also upper bounded by 2 in $2 \mathrm{D}$ random networks.

In [5], authors propose the practical coding system COPE which utilizes intra-flow coding opportunities. In [2], authors propose MORE which utilizes inter-flow coding opportunities. Based on coding scheme in COPE, [11], [12] introduce the coding-aware routing and formulate the max-flow LP with coding considerations, however, they do not incorporate the effect of random access by assuming an optimal link-scheduling for the coding scheme, further study is also necessary on how to realize the coding-aware routing in practice. In [3], authors propose an optimization framework for optimal codingoriented scheduling. In [6], authors propose the first practical coding-aware routing protocol called DCAR. 
This paper falls into the inter-flow coding category, and we analytically examine the coding performance under random access, and shows that the maximum encoding number (which was assumed to be unbounded before) is upper bounded by a constant. Focusing on the coding scheme proposed in [5], we obtain a tighter bound on throughput gain for general wireless networks.

\section{Conclusion}

In this paper, we provide an analysis of the practical coding scheme under realistic physical layer and random access. The key performance measure is the encoding number. We derive an upper bound on the encoding number in any possible coding structures. By calculating the average encoding number, we examine the performance of a general class of random access mechanisms. We also provide a tighter upper bound on the throughput gain by the practical coding scheme. Our analysis can be useful for future coding-related protocol design and analysis [7], in particular, our on-going work include how to quantify the benefit of network coding under moderate to high mobility pattern in wireless ad-hoc networks.

Acknowledgement: the authors would like to thank the reviewers for their valuable comment. This research has been supported in part by the Hong Kong RGC grant 415708, as well as the research grant from the AoE in network coding.

\section{REFERENCES}

[1] R. Ahlswede, N. Cai, S. Li and R. Yeung. Network Informaion Flow. IEEE Transactions on Informaion Theory, 46(4), pp. 1204-1216, July 2000.

[2] S. Chachulski, M. Jennings, S. Katti, and D. Katabi. Trading Structure for Randomness in Wireless Opportunistic Routing. ACM SIGCOMM, pp. 169-180, 2007.

[3] P. Chaporkar and A. Proutiere. Adaptive Network Coding and Scheduling for Maximizing Throughput in Wireless Network. ACM MOBICOM, pp. 135-146, 2007.

[4] D. Couto, D. Aguayo, J. Bicket and R. Morris. A High-Throughput Path Metric for Multi-Hop Wireless Routing. ACM MOBICOM, pp. 134-146, 2003.

[5] S. Katti, H. Rahul, W. Hu, D. Katabi, M. Médard and J. Crowcroft. XORs in the Air: Practical Wireless Network Coding. Proceedings of ACM SIGCOMM, pp. 243-254, 2006.

[6] J. Le, JCS Lui, and DM Chiu. DCAR: Distributed Coding-Aware Routing in Wireless Networks. IEEE ICDCS, pp. 463-469, 2008.

[7] J. Le, JCS Lui, and DM Chiu. DCAR: Distributed Coding-Aware Routing Protocol for Wireless Networks. Accepted for publication in IEEE Transactions on Mobile Computing.

[8] QM Li, DM Chiu and JCS Lui. On the Practical and Security Issues of Batch Content Distribution Via Network Coding. International Conference on Network Protocols, pp. 158-167, 2006.

[9] Z. Li and B. Li. Network Coding: The Case of Multiple Unicast Sessions. Proceedings of Allerton Conference, 2004.

[10] J. Liu, D. Goeckel and D. Towsley. Bounds on the Gain of Network Coding and Broadcasting in Wireless Networks. Proceedings of IEEE INFOCOM, pp. 1658-1666, 2007.

[11] B. Ni, N. Santhapuri, Z. Zhong and S. Nelakuditi. Routing with Opportunistically Coded Exchanges in Wireless Mesh Networks. roceedings of the 2nd IEEE Workshop on Wireless Mesh Networks, pp. 157-159, 2006.

[12] S. Sengupta, S. Rayanchu and S. Banerjee. An Analysis of Wireless Network Coding for Unicast Sessions: The Case for Coding-Aware Routing. IEEE INFOCOM, pp. 1028-1036, 2007.

[13] I. Stojmenovic, A. Nayak, J. Kuruvila, F. Ovalle-Martinez and E. Villanueva-Pena. Physical Layer Impact on the Design and Performance of Routing and Broadcasting Protocols in Ad Hoc and Sensor Networks, Computer Communications, 28(10), pp. 1138-1151, June 2005.
[14] H. Zhai and Y. Fang. Distributed Flow Control and Medium Access in Multihop Ad Hoc Networks. IEEE Transactions on Mobile Computing, 5(11), pp. 1503-1514, 2006.

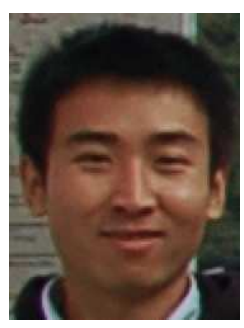

Jilin Le received the B.Eng degree in Electrical Engineering from Peking University, and the M.Phil degree in Computer Science from The Chinese University of Hong Kong. His research interests include wireless networks, network protocols and applications.

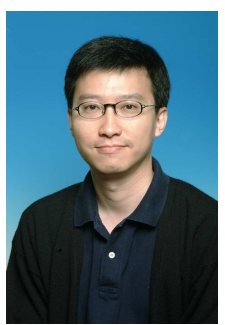

John C.S. Lui received his Ph.D. in Computer Science from UCLA. He is currently the head of the Computer Science \& Engineering Department at the Chinese University of Hong Kong. His research interests span both in systems as well as in theory/mathematics with the emphasis on the robustness, scalability, and security issues on the Internet. John received various departmental teaching awards and the CUHK Vice-Chancellor's Exemplary Teaching Award, as well as the co-recipient of the Best Student Paper Awards in the IFIP WG 7.3 Performance 2005 and the IEEE/IFIP Network Operations and Management (NOMS) Conference. He is an associate editor in the Performance Evaluation Journal, IEEE-TC, IEEE-TPDS and IEEE/ACM Transactions on Networking. John was the TPC co-chair of ACM Sigmetrics 2005 and the General Co-chair for ICNP 2007. John is a fellow of ACM and a fellow of IEEE.

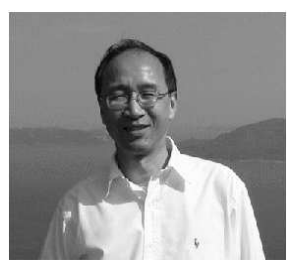

Dah-Ming Chiu received the B.Sc degree in Electrical Engineering from Imperial Collage, University of London, and the Ph.D degree from Harvard University, in 1975 and 1980 respectively. He was a Member of Technical Staff with Bell Labs from 1979 to 1980 . From 1980 to 1996 , he was a Principal Engineer, and later a Consulting Engineer at Digital Equipment Corporation. From 1996 to 2002, he was with Sun Microsystems Research Labs. Currently, he is a professor in the Department of Information Engineering in The Chinese University of Hong Kong. He is known for his contribution in studying network congestion control as a resource allocation problem, the fairness index, and analyzing a distributed algorithm (AIMD) that became the basis for the congestion control algorithm in the Internet. His current research interests include economic issues in networking, $\mathrm{P} 2 \mathrm{P}$ networks, network traffic monitoring and analysis, and resource allocation and congestion control for the Internet with expanding services. Two recent papers he co-authored with students have won best student paper awards from the IFIP Performance Conference and the IEEE NOMS Conference. Recently, Dr Chiu has served on the TPC of IEEE Infocom, IWQoS and various other conferences. He is a member of the editorial board of the IEEE/ACM Transactions on Networking, and the International Journal of Communication Systems (Wiley). He is a fellow of IEEE. 